\title{
LA IMPORTANCIA DE UN BUEN INSTRUMENTO DE EVALUACIÓN: EXPERIENCIA CON LA EVALUACIÓN DE UN VIDEO-TUTORIAL
}

The importance of a good evaluation instrument: experience with the evaluation of a video-tutorial

A importância de umbom instrumento de avaliação: experiênciacomavaliação de um video-tutorial

\section{Pablo Santín Álvarez (1) \\ Zaira Bada Fernández (2)}

(1) CEIP Monte Gándara, El Puente, Zamora. Teléfono: +34 980620233. Correo electrónico: pablo.sanalv.3@educa.jcyl.es

(2) IES Guardo, Guardo, Palencia. Teléfono: +34 979852298. Correo electrónico: zaira.badfer@educa.jcyl.es

\begin{abstract}
Resumen
Esta comunicación analiza la importancia de la evaluación formativa para la mejora del proceso de enseñanza aprendizaje a través de una experiencia realizada con alumnos de $5^{\circ}$ de Educación Primaria. Para ello debían realizar un video-tutorial teniendo como orientación una escala graduada que se entrega por adelantado a los alumnos y que especifica de manera clara los criterios de calificación con los que se va a evaluar el resultado de las producciones de los alumnos. La experiencia demuestra que con el uso de este tipo de instrumentos en procesos de autoevaluación y coevalución no solo se mejoran los resultados de los alumnos, sino que se involucra a todo el alumnado en los procesos de evaluación, haciéndolos partícipes de la misma y convirtiéndolos en los protagonistas de su propio aprendizaje.

Palabras clave: Evaluación formativa; escala graduada; video-tutorial; criterios de calificación
\end{abstract}

\begin{abstract}
This communication analyzes the importance of the formative evaluation for the improvement of the process of teaching learning through an experience realized with students of $5^{\circ}$ of Primary Education. To do this they had to make a video tutorial with a La importancia de un buen instrumento de evaluación: experiencia con la evaluación de un video-tutorial 342
\end{abstract}


graduated scale that is delivered in advance to the students and that clearly specifies the qualification criteria with which to evaluate the results of the students' productions. Experience shows that the use of this type of instruments in self-evaluation and coevaluation processes not only improves students' results, but also engages all students in the assessment processes, making them participants in the same and converting them into the protagonists of their own learning.

Keywords: Formative assessment; graduated scale; video-tutorial; qualification criteria

\section{Resumo}

Esta comunicação discute a importância da avaliação formativa para melhorar o processo de ensino-aprendizagem através de um experimento realizado com estudantes nas classes 5 do ensino primário. Por que tinha que fazer um vídeo tutorial como diretriz uma escala graduada é entregue com antecedência para os alunos e especificando claramente os critérios de qualificação com os quais a serem avaliados os resultados das produções dos alunos. A experiência mostra que o uso de tais instrumentos no processo de auto-avaliação e coevaluación não só os resultados dos alunos são melhoradas, mas todos os estudantes estão envolvidos no processo de avaliação, partilhando com eles o mesmo e transformá-los em os protagonistas de sua própria aprendizagem.

Palavras-chave: Avaliação formativo; escala graduada; vídeo tutorial; critérios de classificação

\section{Introducción}

La evaluación no solo debe ser una herramienta que nos proporcione información sobre si se han conseguido o no los objetivos perseguidos, según Santos-Guerra (2014), esta debe indicarnos también por qué no se han alcanzado. Es aquí donde cobra sentido la evaluación formativa, la cual es entendida por López-Pastor y Pérez-Pueyo, (2017) como el proceso de evaluación cuya principal función es procurar que el alumnado aprenda más y el profesorado perfeccione su práctica docente. No se trata de quedarse en la mera calificación de las producciones de nuestro alumnado, sino de disponer de la información necesaria para que pueda mejorar y aprender más. 
En este sentido, Díaz-Lucea (2005) dice que la evaluación formativa debe estar integrada en el proceso de enseñanza-aprendizaje y en las tareas habituales del profesor. Además tiene que ser una ayuda tanto para los alumnos como para el profesor en vez de suponer una carga añadida.

Como docentes, en ocasiones nos puede producir cierta inquietud que nuestro alumnado obtenga excelentes resultados, ya que se puede pensar que no les presentamos retos a su medida. Pero, ¿acaso no es el sueño de todo docente tener unos alumnos de sobresaliente? Pues permitámosles que puedan llegar a ello dándoles la oportunidad de saber en qué han fallado y qué tienen que hacer para llegar a él. Así seguro que todos, o casi todos, llegarán.

\section{Contextualización}

La experiencia se lleva a cabo en un colegio público de línea 1 de un entorno rural de la provincia de Zamora. La etapa educativa en la que se ha desarrollado es Educación Primaria, concretamente en una clase de $5^{\circ}$ curso que cuenta con 13 alumnos. Se trata de un grupo heterogéneo, algunos de ellos con necesidades específicas de apoyo educativo. El grupo-clase no había tenido ningún contacto con este tipo de evaluación hasta el presente curso.

\section{Diseño y desarrollo}

Antes de comenzar la actividad se deben proporcionar al alumnado los instrumentos de evaluación con unos criterios de calificación claros. Esto les permitirá saber qué demanda la tarea y, una vez entregada, comprobar en qué han fallado para corregirlo y obtener una mejor nota.

Dicho esto, vamos a realizar un análisis de la funcionalidad de la evaluación formativa a través de la realización de un video-tutorial. El objetivo de esta actividad es fomentar la lectura con la realización de un video-tutorial en el que los alumnos recomiendan a otros compañeros un libro que previamente han leído.

Para su elaboración se les entrega a los alumnos una escala graduada (Tabla 1), modificada de Pérez-Pueyo y Casado (2015), con la que desde el primer momento sabrán cómo elaborar dicho tutorial, el contenido que deben incluir en el mismo, y las calificaciones que pueden obtener en función de lo que se adapten a la escala proporcionada. 
Tabla 1.

Escala graduada del video-tutorial adaptada de Pérez-Pueyo y Casado (2015).

\begin{tabular}{|c|c|}
\hline \multirow{6}{*}{ 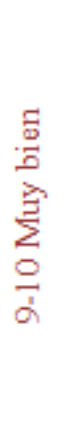 } & $\begin{array}{l}\text { Presentanel conterido solicitado: Título del libro y autor, presentación de los personajes, } \\
\text { breve resumen del libro, recomendación y despedida. }\end{array}$ \\
\hline & Se presentanto dos los aspectos a identificar como el nombre del alumno, centro o curso. \\
\hline & La duración no excede de $2 \mathrm{~min}$. \\
\hline & $\begin{array}{l}\text { Se observa una lógica en el contado y se destacan/resaltan los aspectos importantes a } \\
\text { tener en cuenta. }\end{array}$ \\
\hline & Se sigue el guion preestablecido. \\
\hline & La calidad de la grabación y el cuidado del vídeo son destacables. \\
\hline \multirow{6}{*}{$\begin{array}{l}\stackrel{\Xi}{\vec{\omega}} \\
\stackrel{m}{0} \\
\infty \\
\infty \\
\cdots\end{array}$} & $\begin{array}{l}\text { Presentanel conterido solicitado de manera casi completa (Por ejemplo, se tratan todos } \\
\text { los apartados de un tema aunque en algunos casos de manera superficial). }\end{array}$ \\
\hline & Falta algún elemento por identificar como nombre, curso o colegio. \\
\hline & La duración no excede de $2 \mathrm{~min}$, pero hay partes que sobran. \\
\hline & $\begin{array}{l}\text { En algunos momentos se pierde la lógica en el contado y no se destacan/resaltan aspectos } \\
\text { importantes a tener en cuenta. }\end{array}$ \\
\hline & Se presentó guion pero no se transformó/reflejó en el resultado. \\
\hline & La calidad de la grabación y el cuidado del vídeo son mejorables pero suficientes. \\
\hline \multirow{6}{*}{ 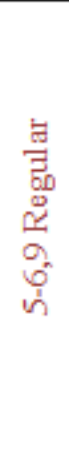 } & $\begin{array}{l}\text { Presentanel conterido de manera incompleta (Por ejemplo, algunos aparta dos del tema } \\
\text { no se tratan). }\end{array}$ \\
\hline & $\begin{array}{l}\text { No se presentan varios de los aspectos a identificar como nombre del alumno, centro o } \\
\text { curso. }\end{array}$ \\
\hline & La duración no excede de $2 \mathrm{~min}$ pero no de $3 \mathrm{~min}$ y hay cosas que sobran. \\
\hline & $\begin{array}{l}\text { No se observa una lógica ni en el contado ni se destacan/resaltan la mayoría } \\
\text { aspectos importantes a tener en cuenta. }\end{array}$ \\
\hline & No se presentó el guion. \\
\hline & La calidad de la grabacióny el cuida do del vídeo se deberían haber trabajado mucho más. \\
\hline \multirow{3}{*}{$\begin{array}{l}\text { J } \\
\sum_{0} \\
0 \\
0 \\
0\end{array}$} & No se presentan el contenido mínimo o la información básica. \\
\hline & La duración supera los $3 \mathrm{~min}$. \\
\hline & No hay explicaciones ni/o comentarios explicativos. \\
\hline
\end{tabular}

Como se puede ver existen cuatro horquillas que van desde el muy bien (9-10) hasta el mal $(0-4,9)$ junto con los respectivos indicadores que acaban siendo los criterios de calificación (Pérez-Pueyo, Hortigüela\& Gutiérrez-García, 2017). El uso de este instrumento con una evaluación formativa, propicia que los alumnos sepan exactamente lo que tienen que mejorar para obtener una mejor nota. Para ello se pueden pactar con los alumnos un número de entregas antes de la calificación final que en este caso fue de tres.

Además el hecho de que sea un instrumento tan objetivo permite que se puedan realizar otros procesos de evaluación como la autoevaluación o coevaluación con éxito La importancia de un buen instrumento de evaluación: experiencia con la evaluación de un video-tutorial 345 
ya en EP (Herranz\& López-Pastor, 2014a);(Herranz\& López-Pastor, 2014b) y evaluación compartida porque los alumnos pueden ver el resultado de su proyección (el video-tutorial) y analizar junto al maestro en qué aspectos debe mejorar teniendo la escala delante, pudiendo incluso parar el vídeo, avanzar o retroceder para comprobar estos aspectos.

Es importante no confundir los criterios de calificación con la porcentualización de la actividad. En ocasiones se le entrega al alumno un documento con la porcentualización de la actividad (Tabla 2) pensando que con ello ya van a ser capaces de realizar un buen trabajo. Sin embargo apenas se le está dando información al alumno para realizar el trabajo.

Tabla 2.

Rúbrica de la descripción de un retrato

\begin{tabular}{|c|c|c|c|c|c|}
\hline $\begin{array}{c}\text { Descripción } \\
1 \mathrm{p}\end{array}$ & $\begin{array}{c}\text { Retrato } \\
1 \mathrm{p}\end{array}$ & $\begin{array}{c}\text { Literario } 2 \mathrm{p} \\
\text { (Sugiere=Connotación) }\end{array}$ & $\begin{array}{c}\text { Extensión } 2 \mathrm{p} \\
(+/- \text { Una Cara })\end{array}$ & $\begin{array}{c}\text { No coloquial } \\
2 \mathrm{p}(-0,25)\end{array}$ & $\begin{array}{c}\text { Sintaxis } 1 \mathrm{p} \\
\text { (Redacción) }\end{array}$ \\
\hline
\end{tabular}

$\mathrm{Al}$ contrario que en esta rúbrica, en la escala graduada que presentamos, sí se puede comprobar cómo el alumno obtiene información relevante sobre lo que puntúa. Además cada indicador o criterio de calificación está secuenciado (Tabla 3) por lo tanto es fácil saber dónde se encuentra la producción del alumno.

Tabla 3.

Ejemplo del mismo criterio secuenciado en las diferentes horquillas

\begin{tabular}{|c|l|}
\hline $9-10$ & $\begin{array}{l}\text { Presentan el contenido solicitado: Título del libro y autor, presentación de los personajes, } \\
\text { breve resumen del libro, recomendación y despedida }\end{array}$ \\
\hline $7-8,9$ & $\begin{array}{l}\text { Presentan el contenido solicitado de manera casi completa (Por ejemplo, se tratan } \\
\text { todos los apartados de un tema aunque en algunos casos de manera superficial). }\end{array}$ \\
\hline $5-6,9$ & $\begin{array}{l}\text { Presentan el contenido de manera incompleta (Por ejemplo, algunos apartados del } \\
\text { tema no se tratan). }\end{array}$ \\
\hline $0-4,9$ & No se presentan el contenido mínimo o la información básica. \\
\hline
\end{tabular}

Para transferirlo a nota numérica, se contabiliza en qué horquilla hay más cruces. Además hay que tener en cuenta el resto de cruces de las otras horquillas. (Tabla 4). El ejemplo posiciona al alumno en la horquilla del 7-8,9, concretamente entre el 7,5 y el 8. Se deja al criterio del profesor la matización del valor final en función de aspectos no contemplados en la escala, como puede ser la creatividad. 
Tabla 4.

Ejemplo de evaluación de escala graduada del video-tutorial

\begin{tabular}{|c|c|c|}
\hline \multirow{6}{*}{ 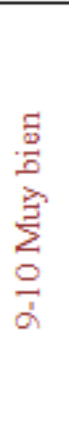 } & & $\begin{array}{l}\text { Presentan el contenido solicitado: Título del libro y autor, presentación de los } \\
\text { personajes, breve resumen del libro, recomendación y despedida. }\end{array}$ \\
\hline & $\mathrm{x}$ & Se presentanto dos los aspectos a identificar como nombre del alumno, centro o curso. \\
\hline & & La duración no excede de $2 \mathrm{~min}$. \\
\hline & & $\begin{array}{l}\text { Se observa una lógica en el contadoy se destacan/resaltan los aspectos importantes a } \\
\text { tener en cuenta. }\end{array}$ \\
\hline & $\mathrm{x}$ & Se sigue el guion preestablecido. \\
\hline & & La calidad de la grabación y el cuidado del vídeo son destacables. \\
\hline \multirow{6}{*}{ 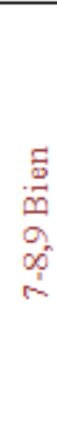 } & $\mathrm{x}$ & $\begin{array}{l}\text { Presentanel conterido solicitado de manera casi completa (Por ejemplo, se tratan todos } \\
\text { los apartados de un tema aunque en algunos casos de manera superficial). }\end{array}$ \\
\hline & & Falta algún elemento por identificar como nombre, curso o colegio. \\
\hline & & La duración no excede de $2 \mathrm{~min}$, pero hay partes que sobran. \\
\hline & $\mathrm{x}$ & $\begin{array}{l}\text { En algunos momentos se pierde la lógica en el contado y no se destacan/resaltan } \\
\text { aspectos importantes a tener en cuenta. }\end{array}$ \\
\hline & & Se presentó guion pero no se transformó/reflejó en el resultado. \\
\hline & $\mathrm{x}$ & La calidad de la grabación y el cuidado del vídeo son mejorables pero sufícientes. \\
\hline \multirow{6}{*}{ 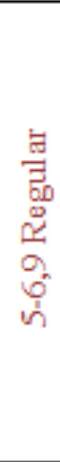 } & & $\begin{array}{l}\text { Presentanel conterido de manera incompleta (Por ejemplo, algunos apartados del tema } \\
\text { no se tratan). }\end{array}$ \\
\hline & & $\begin{array}{l}\text { No se presentan varios de los aspectos a identificar como nombre del alumno, centro o } \\
\text { curso. }\end{array}$ \\
\hline & $\mathrm{x}$ & La duración excede de $2 \mathrm{~min}$ pero no de $3 \mathrm{~min}$ y hay cosas que sobran. \\
\hline & & $\begin{array}{l}\text { No se observa una lógica ni en el contado ni se destacan/resaltan la mayoría } \\
\text { aspectos importantes a tener en cuenta. }\end{array}$ \\
\hline & & No se presentó el guion. \\
\hline & & $\begin{array}{l}\text { La calidadde la grabacióny el cuidado del vídeo se deberían haber trabajado mucho } \\
\text { más. }\end{array}$ \\
\hline \multirow{3}{*}{$\begin{array}{l}\sum_{0}^{\text {J }} \\
0 \\
0 \\
0\end{array}$} & & No se presentan el contenido mínimo o la información básica. \\
\hline & & La duración supera los $3 \mathrm{~min}$. \\
\hline & & No hay explicaciones ni/o comentarios explicativos. \\
\hline
\end{tabular}

\section{Evaluación y conclusiones}

Con la evaluación formativa a través de una escala graduada los alumnos pueden mejorar su trabajo hasta obtener una calificación con la que estén satisfechos. Esta calificación suele ser sobresaliente, pero hay alumnos que se conforman con menos y hay que respetarlo aunque es tarea del maestro convencerlos para que lleguen a desarrollar el máximo de sus capacidades, una de las finalidades de la actual legislación educativa (LOMCE, 2013). 
El hecho de tener claro qué se pide para obtener una buena nota, posibilita que los alumnos tengan la oportunidad de obtenerla.

Además, con la evaluación formativa se consigue que el alumnado se sienta responsable de las notas que obtiene y no lo vea como algo subjetivo que otorga el maestro.

Con la evaluación formativa se consigue mejorar el proceso de enseñanzaaprendizaje, puesto que el alumnado mejora sus producciones y los docentes son conscientes de las necesidades del alumnado, información vital para seguir avanzando con éxito.

\section{Referencias}

Díaz-Lucea, J. (2005). La evaluación formativa como instrumento de aprendizaje en Educación Física. Barcelona: Inde.

Herranz, M., \& López-Pastor, V. M. (2014a). ¿Es viable llevar a cabo procesos de autoevaluación y evaluación compartida en Educación Física en la etapa de Educación Primaria? un estudio de caso longitudinal ( $1^{\text {a }}$ parte). Revista de educación física: Renovar la teoría y práctica, 133, 11-18.

Herranz, M., \& López-Pastor, V. M. (2014b). ¿Es viable llevar a cabo procesos de autoevaluación y evaluación compartida en Educación Física en la etapa de Educación Primaria? un estudio de caso longitudinal ( $2^{\mathrm{a}}$ parte). Revista de educación física: Renovar la teoría y práctica, 133, 29-36.

Ley Orgánica 8/2013, de 9 de diciembre, para la mejora de la calidad educativa (B.O.E., 10 de Diciembre).

López-Pastor, V. M., \& Pérez-Pueyo, Á. (Coords.) (2017). Evaluación formativa y compartida en educación: experiencias de éxito en todas las etapas educativas. León: Universidad de León. Recuperado de http://buleria.unileon.es/handle/10612/5999

Pérez-Pueyo, A., \& Casado, O. M., (2015). Escala graduada para la valoración de un video-tutorial. Recuperado de http://www.grupoactitudes.com/\#!competenciasbsicas-nueva/c1dub 
Pérez-Pueyo, A., Hortigüela Alcalá, D., \& Gutiérrez-García, C. (2017). Reflexión sobre la evaluación en la formación inicial del profesorado en España. En búsqueda de la concordancia entre dos mundos. Revista Infancia, Educación y Aprendizaje, 2(2), 39-75.

Santos-Guerra, M. A. (2014). La evaluación como aprendizaje: Cuando la flecha impacta en la diana. Madrid: Narcea Ediciones. 